\title{
AN ANALYTICAL APPROACH TO THE NEURONAL MECHANISMS UNDERLYING THE MEMORY STORAGE IN THE BRAIN. (EXPERIMENTAL STUDY)
}

\author{
Elias Koutsoukos ${ }^{1,2}$, Elias Angelopoulos², Anthony Maillis ${ }^{1,2}$ \\ and Costas Stefanis ${ }^{1}$
}

(1) University Mental Health Research Institute. Experimental Neurophysiology Laboratory.
Argyrocastrou \& lonias str. P.O.Box 66517, 15601 Papagos, Athens, Greece.

(2) Athens University, Med. School, Dept. of Psychiatry, Eginition Hospital, 74 Vas. Sofias Ave., 11528 Athens Greece.

\begin{abstract}
In the present in vivo experimental study, the dynamical properties of the electroencephalographic (EEG) activity recorded in the CAl area and the dentate gyrous of rat hippocampus were investigated prior to the induction and during the maintenance phase of Long Term Potentiation (LTP). Ourfindings suggest that this form of brain plasticity can be reflected by the complexity of spontaneous EEG activity, as it is expressed by the reduction of the correlation dimension D2, indicates that different functional states of the brain are governed by different degrees of functional complexity.
\end{abstract}

Keywords: Long term potentiation, EEG, (u) theta rhythm deterministic chaos, non linear dynamical analysis, memory processes.

\section{INTRODUCTION}

High-frequency activation of the excitatory synapses in the dentate gyrus and the CA1 region of hippocampus results in an NMDA glutamate receptor dependent, long term increase in synaptic strength referred to as long-term potentiation (LTP) [15]. This use-dependent synaptic plasticity has received considerable attention as a potent neuronal mechanism underlying information storage in the mammalian brain and provides a possible cellular basis for many types of learning and memory. Several recent studies have treated of LTP as a candidate mnemonic mechanism [9]. Electroencephalographic activity (EEG) can be considered either as a stochastic process, or alternatively as a process generated by nonlinear dynamical systems exhibiting chaotic behavior $[2,3,8]$. Following this second conceptualization of deterministic chaos, various states of EEG activity were analyzed in order to approach underlying mechanisms in normal and pathophysiological brain functioning. In the present in vivo experimental study, the dynamical properties of the EEG activity recorded in the CA1 area and the dentate gyrous of rat hippocampus were investigated prior to the induction and during the maintenance phase of LTP.

\section{GENERAL PROCEDURE}

We decided to investigate, for comparison reasons, two hippocampal regions related to the memory processes the CA 1 and the dentate gyrus (DG) by stimulating the CA3 and perforant path (pp) respectively. The experiments were conducted on Wistar rats under chloral hydrate anesthesia (40mg/100g intraperitoneally). In both experimental groups the stimulation and recording electrodes were lowered stereotaxically into the above mentioned areas.

Evoked potentials and spontaneous EEG activity were recorded via the same electrode, using a common headstage amplifier and two separated sets of amplifiers - filters. Both signals were immediately digitized and stored with sampling rates, 10000 and 512 samples / sec respectively. In both experimental groups (pp-DG and cCA3-CA1) dentate gyrus and CA1 area were stimulated with single square wave pulses $(0.05 \mathrm{~Hz}$ and $0.2 \mathrm{~ms}$ duration) and control traces were collected for a time period of $30 \mathrm{~min}$. Following tetanic stimulation (ten 200 $\mathrm{Hz}$ stimulus bursts, pulse duration $0.1 \mathrm{~ms}$ and burst duration $200 \mathrm{~ms}$ ) the development and maintenance of LTP were investigated for up to two hours. For the analog instrumentation the Digitimer (Neurolog series) stimulators, preamplifiers, filters and signal conditioners were used. The stimulation timing protocol and the multiple data acquisition procedure were performed using two Keithley DAS $1202 \mathrm{~A} / \mathrm{D}$ boards. The stimulation and acquisition software modules were developed via CEC TestPoint package. 


\section{COMPUTATIONAL PROCEDURE}

For the dimensionality computations the phase space reconstruction of the recorded EEG activities was made following the Takens proposal [21], which spans the time series in m-dimensional equivalents, using the time-shift method. The choice of the time lag $t$ within $50-80 \mathrm{~ms}$ was made by (1) considering a $t$, approximately equal to $25 \%$ of the signal's pseudo-cycle, (2) evaluating D2 and (3) repeating the calculations for a range of T's around the above considered value in order to find where $D 2$ remains stationary. The Grassberger and Procaccia[10] correlation dimension algorithm (1) was computed for hippocampal EEG epochs, constructed from 9600 data points each. For the selection of the reference points and techniques to avoid underestimation of the correlation dimension (see also ref. [11]). The slope estimation of the formed $\log C(r, m)$ vs. $\log r$ curves were evaluated after isolation of the linear part of the curves and subsequent application of the least square method (figure 1).

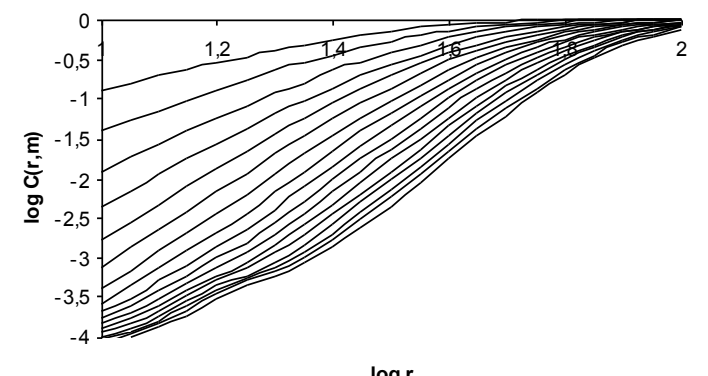

$\log r$

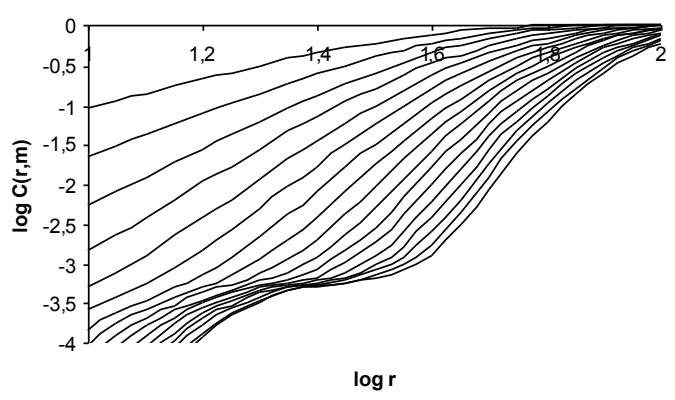

Figure 1. Formation of $\log C(r, m)$ vs $\log r$ curves for embedding dimension up to 18 $(m=18)$. For the left graph the source signal was extracellular EEG activity recorded after the induction of LTP and for the right graph the signal source was the control activity. At the same time the corresponding time series did not show any significant spectral changes.

$$
C(r)=\frac{1}{N^{2}} \sum_{\substack{i, j=1 \\ i \neq j}}^{N} \Theta\left(r-\left|x_{i}-x_{j}\right|\right)
$$

were $\boldsymbol{N}$ - number of points, $\boldsymbol{i}, \boldsymbol{j}$ - arbitrarily selected points of the m-dimensional state space trajectory, $\Theta$ - Heavyside function, $\boldsymbol{r}$-radius.

This widely used procedure reconstructs the de-dimensional state space from the obtained time series and then counts the number of points which lie in a hypercube with radius $r$ around a reference point. This counting is performed for subsequently increased radii $r$ until all the points of the of the formed state space lie within the hypercube. The results of this counting procedure is then averaged for each increment of $r$ and plotted versus $r$ on a double logarithmic scale. The slope of the formed $S$-like curves, considering the straight part of the curve, determines the correlation dimension for the respective embedding dimension $d e$. Thus $C(r)$ behaves like $\sim r^{d}$ and If the plot of correlation dimension $d$ versus de shows a saturation value, this value is taken as the correlation dimension of the attractor.

\section{RESULTS}

Following the single pulse current stimulation of $\mathrm{CA} 3$ region of hippocampus, the $\mathrm{CA} 1$ region responded with a field response known as population spike. This type of activation represents the summated firing from the pyramidal neurons. In figure 2 this evoked response is indicated by the lower amplitude wave. The higher amplitude wave indicates the same cell's response to the same input stimulus following the induction of Long Term Potentiation.

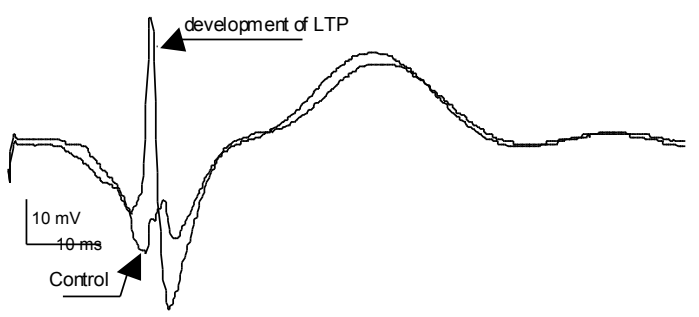

\section{Figure 2. Representative traces of the formed population spike prior and following the development of LTP}

Figure 3 shows the variation of the population spike and EPSP slope after the application of the tetanic stimulation. The observed differences were significant compared to the initial (-30min) values (repeated measurements ANOVA; $\mathrm{df}=5,20, \mathrm{~F}=38.6$, $\mathrm{p}<0.05)$. 


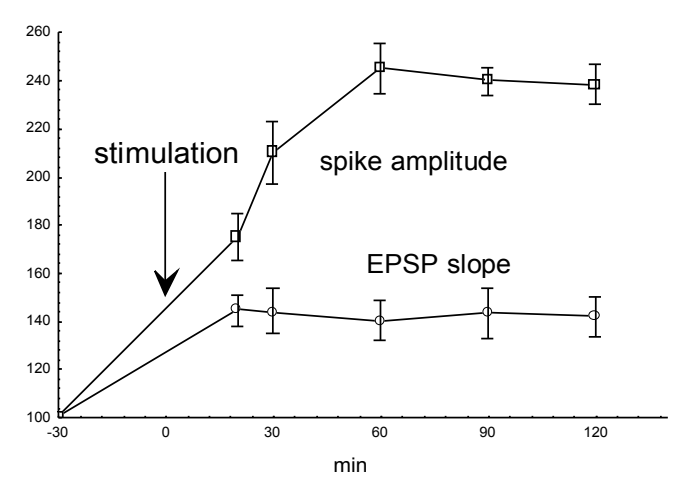

Figure 3. Population spike and slope variation (mean $\pm S D$ ) during the maintenance phase of LTP

The next figure (4) summarizes the results of the dimensionality computation obtained at the same time periods showed a significant maintained decrease of $D 2$, compared to the initial (-30 $\mathrm{min})$ values (repeated measures ANOVA; $\mathrm{df}=5,20$, $\mathrm{f}=6.45, \mathrm{p}<0.05$ for the $\mathrm{pp}-\mathrm{DG}$ group and $\mathrm{df}=5,20$, $\mathrm{f}=6.38 \mathrm{p}<0.05$ for the $\mathrm{cC}$ A3-CA1 group). Post-hoc Sheffe tests showed significant differences $(p<0.05)$ only among control and post tetanus values of D2.

Each separated value of D2, for each experiment and for the specified time period, was obtained as the average of 5 D2's computed from 5 randomly overlapped EEG segments corresponding to the vicinity of the specified stimulus free time zones. In order to exclude possible interference of anesthesia and time effects on quantitative and qualitative pattern of EEG activity five control experiments ( 5 animals) were performed under the same experimental conditions, omitting only the application of the tetanic stimulation. Results from the dimensionality computations of the control group did not show any significant difference in the values of D2, as shown in fig. 1 (repeated measures ANOVA; $\mathrm{df}=5,20, \mathrm{f}=1.42$, $\mathrm{p}=0.25$ ). Post-hoc Sheffe tests were also performed and showed no significant differences. Fast Fourier transformations (FFT's) performed in all experiments at random time intervals, showed no significant differences of power spectra prior and after the establishment of LTP.

In an other part of experiments, the potential role of theta $(\Theta)$ rhythm in the development of LTP was investigated. It is known that $(\Theta)$ rhythm is an sinusoidal extracellularly recorded potential of $5-9 \mathrm{~Hz}$ in the dentate gyrous and CA1 field of hippocampus In the rat occurs during voluntary movement (such as exploration) as well as in REM sleep [25]. The sources and mechanisms of gen- eration of $(\Theta)$ rhythm are unknown but it presumably reflects the action of synchronous postsynaptic potentials resulting in sinusoidal variation of membrane potentials in the hippocampal area $[1,9]$. This type of rhythmic slow activity has found to relate with the development of LTP in the CA1 hippocampal area [18]. In our experiments, under continuous monitoring and spectral analysis, the area of hippocampus that exhibits clear theta was localized. For this experimental condition a separated exploratory microelectrode was stereotaxically lowered in the above brain area. Following the detection and discrimination of $(\Theta)$ rhythm pair trigger pulses were generated (figure 5). The initiation of this bursts was subject of phase correction, since the analog filtering of the signal introduces phase error.

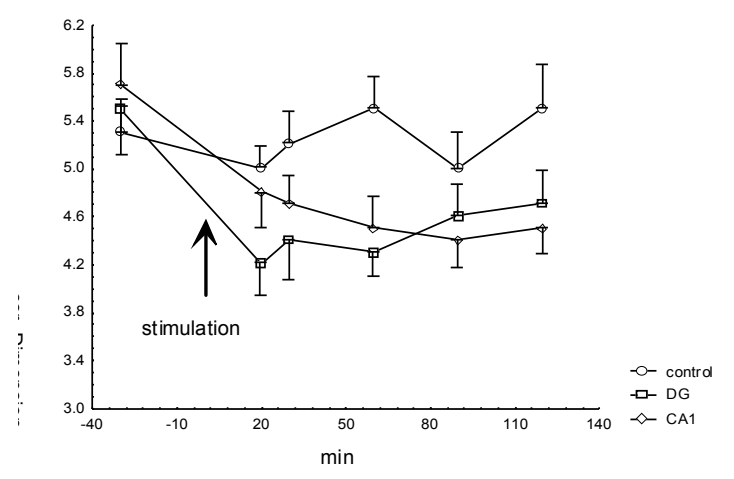

Figure 4. Correlation dimension vs time for control and experimental groups (mean values $\pm S D$ )

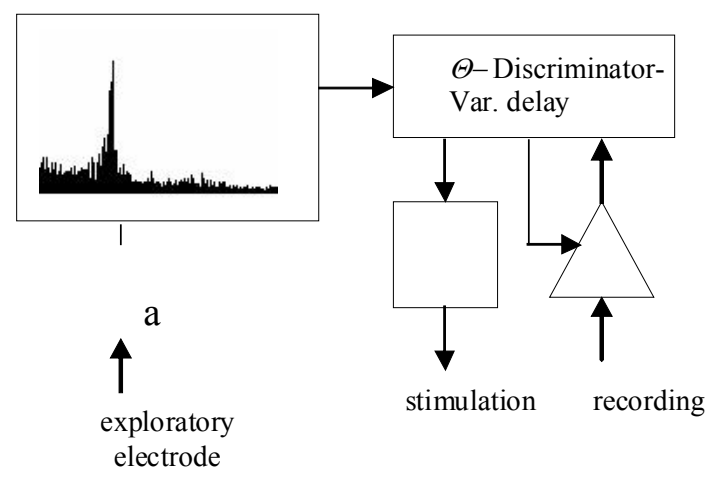

Figure 5. Arrangement for the detection of $(\Theta)$ rhythm and subsequent stimulation of perforant path. a: amplification-filtering, $b$ : stimulator

From our experiments when stimulating train pulses applied on the positive peak of $u$ activity and in a window width of $25 \mathrm{~ms}$ induced LTP, indicating an increase of synaptic efficacy. On the other hand, when stimulation occurred at randomly selected latencies beyond the $25 \mathrm{~ms}$ window, did 
not develop this type of neuronal plasticity. This finding supports that the induction of LTP in the DG area of hippocampus depends preferentially on a specific phase of the locally recorded $u$ rhythm which is used as clock for the synchronization of stimulation.

\section{CONCLUSION}

The hippocampus is proposed to operate as a device responsible for the construction of cognitive maps, representations which capture the spatial layout of an animal's experienced environment [16]. According to the working memory hypothesis $[5,17]$, the primary function of the hippocampus is the formation of temporal / personal associations essential for accurate working memory. Another theoretical view put forward by Teyler and DiScenna (1986) proposes that the main role of the hippocampus is to form and retain an index of neocortical areas activated by experiential events. The hippocampal memory indexing theory claims that the hippocampus stores an index of recently activated cortical loci by the mechanism of LTP. The hippocampal formation comprising the hippocampus proper, dentate gyrus and subiculum has been consistently implicated in information storage models of the mammalian brain $[5,16,17,22]$. Earlier studies [23] suggest that LTP is a possible substrate for long-term information encoding and that interactions between hippocampus and neocortex may play a role in memory functions through the process of LTP. However, the neuronal mechanisms underlying LTP are still unclear. Presynaptic and postsynaptic models have been proposed to explain LTP-associated changes seen on (1) the anatomical $[6,12],(2)$ the physiological [7] and (3) the biochemical level [4].

The induction of LTP during $(u)$ rhythm synchronization, and more specifically within a time window of $25 \mathrm{~ms}$ following the positive phase of recorded wave suggest a new function of this rhythmic slow activity namely a mnemonic function during the behaviours in which it occurs. This finding indicates the potential modulatory role of $(\Theta)$ rhythm in memory storage circuitry.

In this study we applied analytical tools based on recent concepts of deterministic chaos, in order to explore the dynamical properties of LTP underlying mechanisms and their localization in the hippocampus. However several confounding factors have to be considered, particularly when dealing with physiological data which are noise prone and usually non-stationary. The data length of the samples is not optimal and has to be in balance with the stationarity of the signal and the available computational capabilities. The GP algorithmis not ideally suited for the determination of the dimension of high dimensional attractors. Layne et al. (1985) discussed problems associated with dimensional analysis of EEG data and found, even for long data sets 15000 points, high degree of uncertainty of the performed dimensionality calculations. In our study we chose to focus on the comparative sense of the calculated dimensionalities before and after the establishment of LTP, rather than to determine the precise values of the correlation dimension for the two experimental conditions. In spite the above limitations, the dimensionality computation technique appears to be a tool appropriate for the investigation of brain dynamics, particularly when a quantitative descriptor is needed to express the complexity of gross (mass) brain activity.

Our results taken together with previous observations [11], demonstrate that LTP-related changes previously described on the anatomical, physiological and biochemical level, are describable on yet another, fourth level of brain functioning, that of functional complexity. The reduction in the correlation dimension of hippocampal EEG epochs seen during the maintenance phase of LTP was a common finding in both CA1 area and dentate gyrus, indicating the existence of similar processes in both hippocampal areas. This reduction in functional complexity was not accompanied by significant spectral changes, which is in line with observations reported by others $[19,20]$ under different experimental conditions.

Our findings suggest that the physiological mechanisms underlying the neuronal activity may be affected by the plastic neuronal changes occurring during the maintenance phase of LTP in dentate gyrus and CA1 hippocampal area. The fact that this form of brain plasticity can be reflected by the complexity of spontaneous EEG activity, as it is expressed by the reduction of the dimension D2, indicates that different functional states of the brain are governed by different degrees of functional complexity or, in other words, that the increased efficacy in the information process achieved by the induction of LTP modifies the neuronal activity in a plastic manner.

\section{REFERENCES}

[1] P. Andersen. Participating neurons and mechanisms underlying theta activity in urethanized rabbits, Prog. Brain Res 54 (1980). p. 371-380 
[2] A. Babloyantz. Measures of Complexity and Chaos. Editor N.B. Abraham A.L. Albano A. Passamente P.E. Rapp. NATO ARW series, Plenum Press, 1989. p. 51-62

[3] A. Babloyantz. A. Destexhe. Low dimensional chaos in an instance of epilepsy, Proc. Natl. Acad. Sci. USA 83 (1986). p. 3513-3517

[4] M. Baudry. G. Lynch. Hippocampal glutamate receptors, Molec. Cell. Biochem 38(1981). p. 5-18

[5] J.T. Becker. D.S. Olton. Cognitive mapping and hippocampal function, Neuropsychologia 19 (1982). p. 733-741

[6] N.L Desmond. W.B. Levy. Synaptic correlates of associative potentiation depression: an ultrastructural study in the hippocampus, Brain Res 265 (1983). p. 21-30

[7] R,M. Douglas. Long lasting synaptic potentiation in the rat dentate gyrus following brief high frequency stimulation, Brain Res 126 (1977). p. 361-365

[8] W.Freeman. C. Skarda. Spatial EEG patterns, non linear analysis and perception: The neosherringtonian view, Brain Research Review 10 (1985). p. 147-175

[9]Y. Fugita. T. Sato. Inntracellular records from hippocampal pyramidal cells in rabbit during theta rhythm activity, J. Neurophysiol. 27 (1984). p. 1011-1025

[10] P. Grassberger. I. Procaccia. Measuring the strangeness of strange attractors, Physica 9D (1983). p. 1189-1208

[11] E. Koutsoukos. E. Angelopoulos. A. Maillis. C. Stefanis. Dimensionality alterations of the hippocampal electroencephalographic activity following the induction of long-term potentiation in rats, Neuroscience Letters 175 (1994). p. $85-88$

[12] K.S Lee. F.Schottler. M. Oliver. G. Lynch. Brief bursts of high-frequency stimulation produce two types of structural change in the hippocampus, J. Neurophysiol 44 (1980). p. 247-258

[13] S. Layne. G. Mayer-Kress. J. Holzfuss. Dimensions and entropies in chaotic systems. Editor Mayer - Kress G. Springer, Berlin Heidelberg N. York, 1985

[14] W. Lutzenberger. N. Birbaumer. H. Flor. B. Rockstroh, T. Elbert. Dimensional analysis of the human EEG and intelligence, Neuroscience Letters 143 (1992). p. 10-14

[15] R.A. Nicol D.J.A. Wyllie T. Manabe D.J. Perkel. Long term potentiation: A debate of current issues. Editor P. Ascher A.I. Selverston, MIT Press Cambridge, (1994). p. 189-202

[16] J. O' Keefe. L. Nadel. The hippocampus as a cognitive map, Behavioral and Brain Sciences 2 (1979). p. 478-533

[17] D.S.Olton. J.T. Becker. G.E. Handelman. Hippocampal function: Working memory or cognitive mapping?, Physiological Psychology 8 (1980). p. 239-246

[18] C. Pavlides. Y. Greenstein. M. Grudman. $J$. Winson. Long term potentiation in the dentate gyrus induced preferentially on the positive phase of theta rhythm, Brain Research 439 (1988). p. 383-387

[19] J.P. Pijn. J.V. Neerven. A. Noest. F.H. Lopes da Silva. Chaos or noise in EEG signals; dependence on state and brain site, Electroengephalography and Clinical Neurophysiology 79 (1991). p. 371-381

[20] J. Roschke. J.B. Aldenhoff. A non linear approach to brain function: Deterministic chaos and sleep EEG, Sleep 15(2) (1992). p. 95-101

[21] F. Takens. Lecture notes in Mathematics Editor D.A. Rand L.S. Young. Springer New York, 1981. p. 365-381

[22] T.J. Teyler P. DiScenna. Long term potentiation as a candidate mnemonic device, Brain Research Review 7 (1984). p. 15-28

[23] T.J. Teyler. P. DiScenna. The topological anatomy of the hippocampus: A clue to its function, Brain Research Bulletin 12 (1984). p. 711 719

[24] T.J. Teyler. P. DiScenna. The hippocampal memory indexing theory, Behavioral Neuroscience 100 (1986). p. 147-154

[25] C. Vanderwolf. Hippocampal electrical activity and voluntary movement in the rat, Electroencephalogr. Clin. Neurophysiol. 26 (1969). p.407-418

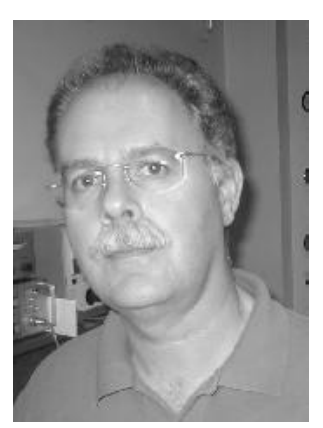

Dr Elias Koutsoukos (born in 1958 in Athens, married, one child) is electronic engineer. He is working as researcher at the Medical School of the University of Athens and at the University Mental Health Research Institute (UMHRI). He is experienced on neurophysiological studies involving intracellular and extracellular recordings of brain neurons with emphasis on the analog and digital signal processing. His current research activity is associated with the application of non linear analytical algorithms on electrophysiological data-sets obtained from brain regions related to the memory processes. This activity has been initiated on the basic research 
level (experimental) giving important results and currently it has been extended on electrophysiological investigations based on healthy humans under memory storage and recall trials. He is also working on the design and development of force balance very broadband seismic sensors, keeping scientific collaboration with national and other institutions abroad.

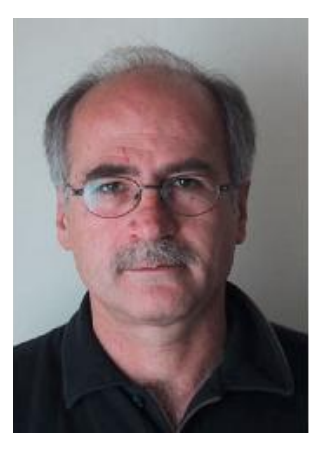

Dr Elias Angelopoulos is a medical doctor and lecturer in psychiatry in the Medical School of the University of Athens. He is born in 1953 in Greece His scientific work includes studies on brain plasticity (opioid induced synaptic plasticity and long-term potentiation).

His current research includes studies regarding brain plasticity and psychopathology on first episode schizophrenic patients. The issues that are addressed in these studies are: (1) time dependent effects of psychotic experience on brain function and (2) how the antipsychotic drugs influense changes in brain plasticity and how these changes are related to the outcome of treatment 